\title{
Perfil ecográfico de madurez pulmonar fetal: quince años de experiencia en el Hospital Nacional Arzobispo Loayza
}

\author{
Alex Alberto Guibovich Mesinas ${ }^{1}$
}

\section{RESUMEN}

Objetivo: Evaluar la capacidad predictiva del Perfil de Madurez Pulmonar Fetal Ecográfico en comparación con el Test de Clements para predecir de manera confiable la Madurez Fetal, evitando las potenciales complicaciones de la amniocentesis, y las limitaciones que hay en nuestro medio para el uso de marcadores bioquímicos de predicción de madurez fetal, como el test de la Lecitina/Esfingomielina, entre otros.

Material y Métodos: estudio descriptivo, prospectivo de evaluación de parámetros diagnósticos para determinar la madurez pulmonar fetal usando como procedimientos la ecografía y la amniocentesis, y comparándolo con el resultado perinatal como patrón de oro. Se estudió 1200 gestantes hospitalizadas en el Servicio de alto riesgo obstétrico del Hospital Arzobispo Loayza desde Diciembre 1995 a Diciembre 2010 a quienes se les realizó el perfil de madurez pulmonar ecográfico (PEMPE) con cinco parámetros: Grado de madurez placentaria, patrón intestinal, relación pulmón-hígado, epífisis femoral distal, y edad gestacional compuesta por biometría múltiple. A todas se les realizó amniocentesis para determinar test de clements (tres tubos) en líquido amniótico. Se evaluó el resultado perinatal al terminar la gestación por indicación obstétrica a más tardar antes de las 48 horas de realizadas las pruebas.

Resultados: Se obtuvieron 1251 RN, ya que hubo 50 embarazos múltiples. El diagnóstico más frecuente fue preeclampsia, la EG por Capurro en promedio fue 34.4 + 1.6 semanas (rango de 27 a 36 semanas), el peso de los RN en promedio fue de 2136 + 580 gr (rango de 1050 a 2840 gr). En total 80 RN presentaron Enfermedad de Membrana Hialina, diagnosticadas por criterios clínicos y radiológicos. La predicción de resultados tanto para el PEMPE, y el test de Clements fueron respectivamente: especificidad $(97.35 \%, 85.74 \%)$, sensibilidad $(86.25 \%, 75.0 \%)$, valor predictivo negativo $(69.0 \%, 26.43 \%)$, valor predictivo positivo $(99.04 \%, 98.04 \%)$.

Conclusión: El PEMPE tiene una alta capacidad predictiva de madurez pulmonar fetal, siendo más específica y sensible respecto al test de Clements, con la ventaja de ser fácilmente repetible y reproducible, es no invasiva, más económica, rápida y puede realizarse ambulatoriamente, prediciendo mejor la madurez que inmadurez pulmonar. (Horiz Med 2015; 15(1): 30-37)

Palabras clave: Madurez Pulmonar Fetal, Ecografía Prenatal. (Fuente: DeCS BIREME).

Ultrasonographic fetal lung maturity profile: fifteen years of experience in Hospital Nacional Arzobispo Loayza

\section{ABSTRACT}

Objective: To evaluate the predictive capacity of Sonographic Fetal Lung Maturity Profile compared to the Clements test to reliably predict the Fetal Maturity, avoiding potential complications of amniocentesis, and constraints that exist in our environment for the use of markers prediction biochemical fetal maturity test as lecithin / sphingomyelin among others. Material and Methods: Descriptive, prospective study evaluating diagnostic parameters to determine fetal lung maturity using ultrasound and amniocentesis procedures, and comparing perinatal outcome as the gold standard. 1200 pregnant women hospitalized with high obstetric risk at Hospital Nacional Arzobispo Loayza from December 1995 to December 2010 were studied. They underwent sonographic profile of lung maturity with five parameters; liver, distal femoral epiphysis, and gestational age composed of multiple biometrics. All had an amniocentesis test to determine clement test in amniotic fluid (three tubes). If, by obstetric indication, pregnancy was ended within 48 hours of testing, perinatal outcome was evaluated. Results: 1251 newborns were studied, as there were 50 multiple pregnancies. The most common diagnosis was preeclampsia. Average gestational age by Capurro was $34.4+1.6$ weeks (range 27-36 weeks), the weight of the newborns, in average, was $2136+580$ gr (range 1050-2840 g). In total 80 newborns presented Hyaline Membrane Disease, diagnosed by clinical and radiological criteria. The prediction results for both sonographic fetal lung maturity and Clements test were: specificity $(97.35 \%, 85.74 \%)$, sensitivity $(86.25 \%, 75.0 \%)$, negative $(69.0 \%, 26.43 \%)$ predictive value, positive predictive value $(99.04 \%$, 98.04\%) respectively.

Conclusion: Sonographic fetal lung maturity has a high predictive ability of fetal lung maturity, to be more specific and sensitive relative to Clements test, with the advantage of being easily repeatable and reproducible, it is noninvasive, fast, cheaper and can be done as an outpatient, predicting lung maturity better. (Horiz Med 2015; 15(1): 30-37)

Key words: Injuries, Injuries by Domestic Violence, Forensic examination. (Source: MeSH NLM).

1 Ginecólogo-Obstetra del Hospital Nacional Arzobispo Loayza. Profesor de Medicina de la UNMSM. Lima - Perú 


\section{INTRODUCCIÓN}

Se puede definir la maduración fetal como el proceso por el cual, el feto, alcanza un desarrollo suficiente de sus aparatos y sistemas que le permite un funcionamiento adecuado y la capacidad de adaptación inmediata a la vida extrauterina (1).

Este proceso de adaptación es más crítico en el caso de los pulmones. El desarrollo pulmonar fetal es un proceso que comprende varias fases. La fase alveolar, la más importante, suele comenzar a la 24va semana de amenorrea y se extiende hasta la vida postnatal (2). Durante este periodo, las células alveolares secretan fosfolípidos (surfactante), que desempeñan un papel muy importante en la integridad funcional del pulmón fetal en el momento del parto. Si estos fosfolípidos no están presentes en cantidad suficiente en el momento del nacimiento, los pulmones fetales se colapsarán y el feto recibirá una oxigenación inadecuada. Esta forma de problema se denomina síndrome de dificultad respiratoria (SDR) o, específicamente, enfermedad de la membrana hialina. Por lo general, esta entidad afecta a fetos prematuros.

El surfactante pulmonar es una mezcla compleja de fosfolípidos y proteínas. El surfactante maduro del adulto esta constituido casi por el $80 \%$ de fosfolípidos, $10 \%$ de proteínas y $10 \%$ de lípidos neutros (3-5).

Hay dos situaciones clínicas importantes en la que es útil contar con una evaluación precisa de la madurez pulmonar fetal in útero dado que ambas pueden provocar un SDR secundario: pretérmino con alto riesgo de parto inminente por trabajo de parto prematuro o en el que se impone el parto pretérmino por indicaciones fetales o maternas (Ej. embarazo de 34 sem con preeclampsia severa y retardo de crecimiento) y embarazo no complicado con fechas desconocidas en el que es necesario una cesárea $(1,6-8)$.

Antes que existieran las pruebas de madurez pulmonar fetal se calculaba que el síndrome de distres respiratorio del recién nacido afectaba a casi 3000 productos al año en Estados Unidos con tasa de mortalidad de casi $30 \%$. Uno de los avances más importantes en la atención perinatal de embarazos de alto riesgo ha sido el descubrimiento y la disponibilidad de una prueba valida y confiable para estimar el grado de madurez pulmonar del feto humano dentro del útero. La aparición de pruebas bioquímicas para predecir la inmadurez pulmonar fetal pudo realizarse sólo después que se dilucido la fisiopatología del $\operatorname{SDR}(9,10)$.

Como el pulmón fetal en desarrollo secreta fosfolípidos surfactantes que llegan al líquido amniótico por los movimientos fetales intra útero, se originó el concepto que mediante el muestreo y cuantificación de estos diversos fosfolípidos $(11,12,13)$, mediante amniocentesis se podía inferir el estado del pulmón fetal y subsecuentemente predecir la aparición de SDR en el periodo neonatal.

En 197l, Gluck y Kulovích (2) demostraron que en embarazos considerados al azar tanto normales como anormales, cuando la relación lecítina/ esfingomielina era de 2 o mayor, no había SDR en el momento del nacimiento, independiente de la edad y el peso $(3,14,15)$.

Los refinamientos en la evaluación de los fosfolípidos en el líquido amniótico para la determinación de la madurez pulmonar fetal han determinado un perfil pulmonar fetal completo $(4,5,16,17)$, que consiste en la relación L/E y los porcentajes de lecitina, fosfatidilinositol y fosfatidilglicerol desaturados (precipitados con acetona). De las 34 a las 37 semanas, hay una fase de transición en la que caben diversos grados de madurez pulmonar fetal. La experiencia clínica ulterior ha indicado que el perfil pulmonar predice la madurez pulmonar del feto con más exactitud que la inmadurez; es decir, se observa virtual ausencia de SDR en fetos con un perfil pulmonar maduro mientras que algunos fetos con perfil pulmonar inmaduro tampoco presentan SDR en el momento del parto (18-20).

Clements y colaboradores (6) vieron que la prueba de agitación era comparable con la razón L/E y tenía un alto valor predictivo de SDR cuando se aplica al líquido amniótico no contaminado. Es más, esta prueba puede utilizarse con entrenamiento y recursos 
mínimos. La prueba de agitación tiene el potencial de ser una prueba de detección rápida, que puede realizarse en 30 minutos. Sin embargo, un resultado inmaduro o transicional de esta prueba sobrestima el riesgo de presentar SDR y debería verificarse mediante cuantificación de la razón L/E (7-9).

La amniocentesis practicada bajo guía ecográfica suele ser un procedimiento benigno (2, 21-24). Por ser invasivos, tienen riesgos, en mínima proporción, pero serios: desprendimiento prematuro de placenta, ruptura prematura de membranas, trabajo de parto prematuro, sangrado fetal o materno, incluso muerte fetal y generan ansiedad materna. Hay limitaciones técnicas en nuestros servicios, escasa cantidad de equipos, agujas de calibre inadecuado e insumos (alcohol) no preservadas de manera adecuada, alterando los resultados, (25-27).

Debido a las potenciales complicaciones de la amniocentesis y las limitaciones que hay en nuestro medio para el uso de marcadores bioquímicos, se estima conveniente realizar esfuerzos para utilizar la ecografía prenatal diagnóstica como un medio para avalar la madurez pulmonar fetal como lo plantea Salman y Quetel en 1986 en la reunión anual del American Institute of Ultrasound in Medicine (10) donde plantea un sistema de puntaje (score) de múltiples parámetros para evaluar la madurez pulmonar fetal.

Hasta ahora se ha venido utilizando la amniocentesis para determinar si un feto está o no maduro para un posible parto temprano. Si bien es cierto que éste método ha resultado satisfactorio, no está exento de los efectos secundarios médicos y emocionales que resultan de los métodos invasivos $(29,30)$. Por tal razón surge la necesidad de encontrar nuevas alternativas no invasivas que sean igualmente satisfactorias sin el agregado que si invasivas sugieren.

Así surge el Perfil Ecográfico de Madurez Pulmonar Fetal que al parecer tiene resultados parecidos con la amniocentesis (31). Este enfoque tiene considerable interés teórico porque toma en cuenta muchos parámetros ecográficos (cinco) que, cuando son usados solos, han demostrado tener alguna relación con la madurez pulmonar fetal. Sin embargo, para que gane amplia aceptación, es preciso hacer una evaluación de este sistema en un estudio prospectivo de series de pacientes de modo que se pueda fomentar su aplicación en nuestros hospitales como un medio alternativo (no invasivo, barato y rápido) de diagnóstico de madurez pulmonar fetal (32-34).

El objetivo del presente trabajo fue evaluar el sistema de puntaje múltiple para predecir madurez pulmonar fetal por ecografía (PEMPE) y compararlo con la determinación bioquímica de surfactante (test de clements, e índice de creatinina) en líquido amniótico obtenido por amniocentesis (prueba estándar) y con el resultado perinatal (prueba de oro). Determinando la sensibilidad, especificidad y el valor predictivo positivo y negativo de dichas pruebas, y si existe diferencia estadísticamente significativas entre ellas.

\section{MATERIAL Y MÉTODOS}

Estudio descriptivo, prospectivo de evaluación de pruebas diagnósticas con tipo de muestreo intencional y universal de aquellos pacientes en quienes se hizo necesario realizar una prueba de madurez pulmonar para terminar gestación. La evaluación incluyó una anamnesis dirigida además de datos generales de filiación y el diagnóstico con que ingresó al estudio.

Se evaluaron a todas las gestantes hospitalizadas en el Servicio de Obstetricia del Hospital Arzobispo Loayza desde Diciembre de 1995 a Diciembre del 2010, de 27 a 36 semanas de edad gestacional por última regla, con o sin patología, quienes se sometieron a una prueba de amniocentesis y perfil ecográfico para valorar madurez pulmonar fetal. Las patologías incluyeron los casos donde fue necesario realizar una prueba de madurez pulmonar para terminar gestación, por ejemplo, casos de preeclampsia leve, verificación de edad gestacional por error de fecha de última regla (FUR), retardo de crecimiento intrauterino (RCIU) amenaza de parto pretérmino (APPT), cesareada previa sin condiciones para chance vaginal, gestantes normales sin patología quienes requerían por alguna 
circunstancia determinar características del líquido amniótico y determinar si existía madurez pulmonar cuando tuviera edad gestacional calculada mayor de 26 semanas y menor de 37 semanas.

La amniocentesis fue realizada utilizando la técnica estéril estándar inmediatamente después de haberse probado el examen ecográfico del perfil de madurez pulmonar fetal.

Fueron estudiadas 1200 pacientes gestantes a quienes se les realizaron amniocentesis. Todos los partos ocurrieron dentro de las 48 horas después de la última amniocentesis.

\section{Descripción de las variables de estudio}

Se realizó la captación de datos a través de la evaluación ecográfica y de amniocentesis.

El líquido amniótico obtenido mediante amniocentesis fue por indicación médica.

Se evaluaron las siguientes variables:

Test de Clements, el líquido amniótico obtenido mediante amniocentesis fue sometido a la prueba de los tres tubos. La prueba fue considerada positiva si el test mostraba espuma en 2 tubos.

No se procesaron las muestras contaminadas con sangre o meconio.

Se usó un ecógrafo marca Aloka con transductor lineal convexo de 3,5 $\mathrm{MH}$ y se evaluaron la combinación de los cinco parámetros planteados en el sistema de Salmán y Quetel (10).

Se estableció un score de puntaje y se consideró inmaduro si el puntaje fue de 0-4 y maduro si el puntaje fue de 5-10. Tabla1.

DBP diámetro biparietal; PC, perímetro cefálico: PA, perímetro abdominal; LF, longitud del fémur.

Reproducido de Salman D. Quetel T. Sonographic scoring of fetal Pulmonar y maturity J. Ultrasound Med. 5 (Suppl): 145. 1985.

Se evaluó y comparó los resultados de la ecografía y la amniocentesis con el resultado perinatal.
Tabla 1. Sistema de Puntaje Compuesto para evaluar madurez pulmonar

\begin{tabular}{|c|c|}
\hline $\begin{array}{c}\text { Mediciones y Observaciones } \\
\text { Ecográficas }\end{array}$ & $\begin{array}{l}\text { Puntaje de } \\
\text { Madurez Pulmonar }\end{array}$ \\
\hline \multicolumn{2}{|l|}{ Edad compuesta (por DBP, PC, PALF) } \\
\hline (Hadlock y col. L6) & 0 \\
\hline$<35$ sem & 1 \\
\hline$>37$ sem & 2 \\
\hline \multicolumn{2}{|l|}{ Grado placentario (Grannum y col. 15) } \\
\hline $0-1$ & 0 \\
\hline$\|$ & 1 \\
\hline III & 2 \\
\hline \multicolumn{2}{|c|}{ Patrón del intestino fetal (Zilianti y Fernández. 17) } \\
\hline Estadios 1 - 2 & 0 \\
\hline Estadios 3 & 1 \\
\hline Estadio 4 & 2 \\
\hline \multicolumn{2}{|l|}{ Relación pulmón/hígado (Morris. 18) } \\
\hline$<1$ & 0 \\
\hline$=1$ & 1 \\
\hline$>1$ & 2 \\
\hline \multicolumn{2}{|l|}{ Epífisis femoral distal (Chinn y col. 19) } \\
\hline Ausente & 0 \\
\hline Tipo hendidura & 1 \\
\hline Globular & 2 \\
\hline
\end{tabular}

El resultado perinatal (evolución neonatal) incluyó dos respuestas:

Bueno: RN normal sin patología respiratoria. Malo: RN con distres respiratorio debido a EMH.

Se evaluó la causa del distres respiratorio sobre la base de la historia clínica, el examen físico y el estudio radiológico pulmonar. Los síntomas clínicos del distrés usados en la evaluación del paciente incluyeron: taquipnea (mayor de 60 por minuto), cianosis, retracciones subcostales y la necesidad de suplemento de oxigeno con $\mathrm{cl}$ fin de mantener una P02 por encima de $50 \mathrm{~mm} \mathrm{Hg}$. Para hacer diagnóstico de enfermedad de membrana hialina EMH el RN tuvo que presentar criterios clínicos y radiológicos sugestivos de EMH.

RN con distres respiratorio debido a neumonía o a enfermedad cardiaca congénita fueron excluidos del diagnóstico de EMMH y se consideró para efectos de este estudio dentro del grupo de buen resultado perinatal. 


\section{Análisis de datos}

Se realizaron dos fases de análisis: univariado en el que se presentan frecuencias y porcentajes para las variables discretas como también las medias y desviaciones estándar para las; variables continúas y la búsqueda de valores de sensibilidad, especificidad y valores predictivos positivos y negativos de los parámetros ecográficos del perfil de madurez pulmonar fetal y de los marcadores bioquímicos del líquido amniótico.
La Tabla 3, resume los resultados finales maternos $y$ fetales.

Las Tablas 4 y 5 , muestran el resultado de la determinación de la madurez pulmonar fetal por ecografía, y por test de Clements, en relación al resultado perinatal.

El PEMPE predijo 69 verdaderos casos de inmadurez, y 1140 casos verdaderos de madurez pulmonar fetal. Hubo 11 casos de falso negativo y 31 casos de falsos positivos. El Test de Clements predijo 60

Tabla 2 Diagnóstico de las gestantes incluidas en estudio

\begin{tabular}{|lll|}
\hline \multicolumn{1}{c}{ DIAGNÓSTICOS } & & \\
\hline PREECLAMPSIA & 540 & 45.00 \\
\hline CESAREADA ANTERIOR & 310 & 25.83 \\
\hline AMENAZA DE PARTO PREMATURO & 140 & 11.66 \\
\hline RESTRICCIÓN DE CRECIMIENTO INTRAUTERINO & 130 & 10.82 \\
EMBARAZO MÚLTIPLE & 50 & 4.16 \\
PLACENTA PREVIA & 30 & 2.50 \\
\hline TOTAL GESTANTES & 1200 & 100.00 \\
TOTAL RECIEN NACIDOS & 1251 & 100.00 \\
\hline
\end{tabular}

Tabla 3 Resultados finales materno- fetales

\begin{tabular}{|llc|}
\hline VARIABLES & & RESULTADOS \\
\hline EDAD MATERNA & Promedio & $26.14+/-5.8$ \\
& Rango & $13-43$ \\
\hline VIA DE PARTO & Cesárea & 98 \\
EDAD GESTIONAL POR CAPURRO & Vaginal & 22 \\
& Promedio & $34.4+/-1.6$ \\
& Rango & $27-36$ \\
\hline PESO DEL RECIEN NACIDO & Promedio & $2136+/-580$ \\
APGAR DEL RECIEN NACIDO & Rango & $1050-2840$ \\
& Promedio & $7.02+/-1$ \\
CASOS DE MEMBRANAS HIALINAS & Rango & 4 a 9 \\
& SI & 80 \\
& NO & 1171 \\
\hline
\end{tabular}

\section{RESULTADOS}

Se evaluaron 1200 gestantes, a todas ellas se les realizó amniocentesis y perfil ecográfico de madures pulmonar fetal PEMPE dentro de las mismas 48 horas antes del parto. Los diagnósticos de las madres gestantes se muestran en orden de frecuencia en la Tabla 2. casos verdaderos de inmadurez fetal y 1004 casos verdaderos de madurez pulmonar fetal. Hubo 20 falsos negativos y 167 falsos positivos.

La sensibilidad, especificidad y valores predictivos positivos y negativos obtenidos por el PEMPE y el test de Clements para precisar madurez pulmonar fetal se observa en la Tabla 6. 
Tabla 4 Resultado de determinacion de la madurez pulmonar fetal por ecografia (pempe) y su relacion con el resultado perinatal (presencia o no de membranas hialinas)

\begin{tabular}{|c|l|c|c|c|}
\hline \multicolumn{2}{|c|}{} & \multicolumn{2}{|c|}{ MEMBRANAS HIALINAS (MH) } & \multirow{2}{*}{ TOTAL } \\
\cline { 3 - 4 } \multicolumn{2}{|c|}{} & SI & NO & \multicolumn{1}{|c|}{} \\
\hline $\begin{array}{c}\text { SCORE } \\
\text { ECOGRAFICO }\end{array}$ & INMADURO & $\mathbf{6 9}(\mathbf{6 9 . 0} \%)$ & $31(31.0 \%)$ & $100 \quad(8.00 \%)$ \\
\cline { 2 - 4 } & MADURO & $11(1.0 \%)$ & $\mathbf{1 1 4 0 ( 9 9 . 0 \% )}$ & $1151(92.00 \%)$ \\
\hline \multicolumn{2}{|c|}{ TOTAL } & $80(6.4 \%)$ & $1171(93.6 \%)$ & $1251(100.00 \%)$ \\
\hline
\end{tabular}

Tabla 5 Resultado de determinacion de madurez pulmonar según test de clements y su relacion con el resultado perinatal (presencia o no de membranas hialinas)

\begin{tabular}{|c|l|c|c|c|}
\hline \multicolumn{2}{|c|}{} & \multicolumn{2}{|c|}{ MEMBRANAS HIALINAS (MH) } & \multirow{2}{*}{ TOTAL } \\
\cline { 3 - 4 } \multicolumn{2}{|c|}{ SI } & NO & \\
\hline $\begin{array}{c}\text { TEST DE } \\
\text { CLEMENTS }\end{array}$ & INMADURO & $\mathbf{6 0}(\mathbf{2 6 . 4 4 \% )}$ & $167(73.56 \%)$ & $227(18.15 \%)$ \\
\cline { 2 - 4 } & MADURO & $20(1.96 \%)$ & $1004(\mathbf{9 8 . 0 4} \%)$ & $1024(81.85 \%)$ \\
\hline \multicolumn{2}{|c|}{ TOTAL } & $80(6.40 \%)$ & $1171(93.6 \%)$ & $1251(100.00 \%)$ \\
\hline
\end{tabular}

Tabla 6 Sensibilidad, especificidad, valor predictivo positivo y negativo del pempe, y del test de clements para precisar madurez pulmonar fetal

\begin{tabular}{|c|c|c|c|c|c|c|c|}
\hline & & \multicolumn{2}{|c|}{$\begin{array}{c}\text { MENBRANAS } \\
\text { HIALINAS }\end{array}$} & \multirow{2}{*}{$\begin{array}{c}\text { Especificidad } \\
\text { Sp }\end{array}$} & \multirow{2}{*}{$\begin{array}{l}\text { Sensibilidad } \\
\text { Sb }\end{array}$} & \multirow{2}{*}{ VP + } & \multirow{2}{*}{ VP - } \\
\hline & & SI & NO & & & & \\
\hline \multirow{2}{*}{$\begin{array}{c}\text { SCORE } \\
\text { ECOGRAFICO } \\
\text { (PEMPE) }\end{array}$} & INMADURO & 69 & 31 & \multirow{2}{*}{$97.35 \%$} & \multirow{2}{*}{$86.25 \%$} & \multirow{2}{*}{$99.04 \%$} & \multirow{2}{*}{$69.00 \%$} \\
\hline & MADURO & 11 & 1140 & & & & \\
\hline \multirow{2}{*}{$\begin{array}{c}\text { TEST DE } \\
\text { CLEMENTS } \\
\text { (AMNIOCENTESIS) }\end{array}$} & INMADURO & 60 & 167 & \multirow{2}{*}{$85.74 \%$} & \multirow{2}{*}{$75.00 \%$} & \multirow{2}{*}{$98.04 \%$} & \multirow{2}{*}{$26.43 \%$} \\
\hline & MADURO & 20 & 1004 & & & & \\
\hline
\end{tabular}

Kappa indica hay concordancia deficiente $(<0.4)$ entre ambas pruebas respecto a riesgo de membranas hialinas.

\section{DISCUSIÓN}

La prematuridad iatrogénica y el síndrome de distres respiratorio (SDR) son los factores de morbilidad y mortalidad neonatal asociada a un parto efectivo $(20,21,34)$.

La morbilidad y mortalidad asociadas a un parto prematuro pueden ser reducidas con el uso apropiado de las pruebas de madurez fetal antes de proceder al parto de manera efectiva (22, 35). La prueba más ampliamente utilizada para la evaluación de la madurez fetal es la relación L/E del líquido amniótico (prueba estándar).

Aunque este método es confiable y seguro de predicción de madurez pulmonar fetal $(22,23)$, tiene algunas desventajas: requiere realizar amniocentesis, que debido a su naturaleza invasiva tiene complicaciones fetales y maternas $(24,25,36)$, ocasionalmente, puede fallar debido a oligohidramnios, obesidad materna, localización anterior de la placenta y a contaminación con sangre o meconio.

El estudio ecográfico (un método no invasivo) puede predecir indirectamente la madurez pulmonar fetal, evaluando ciertos parámetros ecográficos y proporcionando valiosa información con mínimo riesgo a la madre y al feto $(26,37-40)$.

En 1986, en la reunión anual del América Institute of Ultrasound in Medicine, Salman y Quetel (10) describieron un sistema de score de puntuación 
(Tabla 1), que con un puntaje de 5 o más, de un total posible de 10, indicaba madurez pulmonar fetal en su grupo de estudio inicial de 104 pacientes.

En nuestro estudio con 1200 pacientes, se encontró que un score mayor o igual a 5 correlaciona con madurez pulmonar fetal en un $97.35 \%$, lo cual implica madurez fetal, y que la posibilidad de distrés respiratorio es mínima.

El PEMPE muestra una mejor especificidad $(97.35 \%$ versus $85.74 \%$ ) y una mejor sensibilidad $(86.25 \%$ versus $75 \%$ ) que el Test de Clements.

En el valor predictivo positivo (predecir verdaderos casos de madurez pulmonar), no hay diferencias significativas entre el PEMPE y el Test de Clements, lo que indica que ambas pruebas son buenas y equivalentes en ello.

En cuanto al valor predictivo negativo (predecir verdaderos casos de inmadurez fetal), el PEMPE es mucho mejor que el Test de Clements, ya que se equivoca menos.

En conclusión, el perfil ecográfico de madurez pulmonar fetal (PEMPE) tiene mejor especificidad, sensibilidad, y valores predictivos positivo y negativo que el test de Clements.

\section{REFERENCIAS BIBLIOGRÁFICAS}

1. Hadlock FP, Irwin JF, Roecker E, et al: Ultrasound prediction of fetal lung maturity. Radiology 155:469, 1985.

2. Gluck L, Kulovich MV. Bprer RD, et al: Diagnosis of the respiratory distress syndrome by amniocentesis. Am J Obstet Gynecol 1991;109:440.

3. Gluck L, Kuluvich MV: Lecithin-spbingomvelin ratios in amniotic fluid in normal and abnormal pregnancy. Am J Obstet Gynecol 1973;15:539.

4. Kulovich MV, Hallman MB, Gluck L: The lung profile I. Normal pregnancy. Am J Obstet Gynecol 1979;135:57.

5. Kulovich MV, Gluck L. The lung profile II, Complicated pregnancy. Am J Obstet Gynecol 1979; 135:64-70.

6. Clements JA. Platzker ACG. Tierney DG, et al. Assessment of the risk of the respiratory distress syndrome by a rapid test for surfactant in amniotic fluid, Am J Obstet Gynecol 1972;286:10771081.
7. Goldstein AS, Fukunaga K, Malachowsky N, et al. A comparison of the lecithin/sphingomyelin ratioand shake test for estimating fetal pulmonary maturity. Am J Obstet Gynecol 1974; 118: 12321135.

8. Sher G, Stantlad BE. Freer DE, et al Assessing fetal lung maturation by the lumadex foam stability index test. Am J Obstet Gynecol 1978;52:673-677.

9. Sher G, Stantlad BE, Assessment of f'etal pulmonary maturity by the lumadex foam stability index test. Am J Obstet Gynecol 1993;61:444-449.

10. Salma F, Quetel T: Sonografic scoring of the pulmonie maturity. J Ultrasound Med 1985; 5 (suppl):145.

11. Collaborative Group on Antenatal Steroid Theraphy: Effect con antenatal dexamethasone administration on the prevention of respiratory distress syndrome. Am J Obstet Gynecol 1981;141:276.

12. Donald IR, Freeman RK, Goebelsmann U, et al: Clinical experience with the amniotic fluid lecithin sphingomyelin ratio: I. Antenatal prediction of fetal lung maturity profile. Obstet Gynecol 1973;115:547.

13. Mackenna J, Hodson CA, Brame RG: Clinical Utility of fetal lung maturity profile. Obstet Gynecol 1981;57:493.

14. Whittle MJ, Wilton AL, Whitefield $C R$, et al: Amniotic fluid phosphatidylglicerol and the lecithin/sphingomyelin ratio in the assessment of fetal lung maturity. $\mathrm{Br} \mathrm{J}$ Obstet Gynecol 1982;89:727.

15. Grannum PAT, Berkovits RL, Hobbins JC: Ultrasonic changes in the maturing placenta and their relation to fetal pulmonic maturity. Am J Obstet Gynecol 1979;133: 915.

16. Hadlock FP. Dter RL. Harris RB: Estimating fetal age: Computerassisted analysis of multiple fetal growth parameters. Radiology 1987;152:497.

17. Ziliantí M Fernandez S: Correlation of ultrasonic images of fetal intestine with gestacional age and fetal maturity. Obstet Gynecol 1983;62:569.

18. Morris SE: Ultrasound: A predictor of' fetal long maturity. Med Ultrasound 1984; 8:1

19. Chinn DH. Bolding BD, Callen PW, et al: Ultrasonographic identification of fetal lower extremity epiphyseal ossification centres. Radiology 1983;147:815.

20. Keettek, W. C. Randall, JH., and Donnelly, M.M.: The hazards of elective induction of labor. Am J Obstet Gynecol 1958;75:496.

21. Goldenberg, R.L., and Nelson, K.: latrogenic respiratory distress syndrome, Am J Obstet Gynecol 1975;123:617.

22. Gluck L, Kulovich MV, Borer R.D, et al: Diagnosis of the. respiratory distress syndrome by amniocentesis. Am J Obstet Gynecol 1971;109:440.

23. Guck L, Kulovich MV, Borer, R.C., ct al: The interpretation and significance of the lecithin-sphingomyelin ratio in amniotic fluid. Am J Obstet. Gynecol. 1974;120:142.

24. Schwartz, R.H.: Amniocentesis, Clin. Obstet. Gynecol 1975; 18:1.

25. Young, B.K.: Report on tirad trimestre amniocentesis at Bellevue 
hospital of New York University Medical Center. New York, in antenatal Diagnosis Repor f a Concensus Development Conference, Bethesda. Maryland, March 5-7, 1979, National Institutes of Health; $11-65$.

26. Campbell, S: The prediction of fetal lung maturity by ultrasound measurement of the biparietal diameter. J Obstet. Gynecol. Br Commonw 1989; 76: 603.

27. Sabbagha, R.E., Turner, J. Rockehe, H., et al: Sonar biparietal diameter an fetal age. Obstet. Gynecol 1974;43:7.

28. Petrucha RA, Golde ST. Plantt LD: Real-time ultrasound of the placenta in assessment of fetal pulmonic maturty. Am J Obstet 1982;142:463.

29. Harman CR, Manning FA, Sterns E. Et al: The correlation of' ultrasonic placenta grading and fetal pulmonary maturation in five hundred sixty-three pregnancies. Am J Obstet. Gynecol 1982;143:941.

30. Quinlan RW, Cruz AC, Buhi WC, et al: Changes in placental ultrasonic apperance: Ineidence of grade III changes in The placenta in correlation to fetal pulmonary maturity. Am J. Obstet Gynecol 1982;144:468.

31. Hills D, Tuck S, Irwin GAL: The unrealiability of placental gradings as an indicator of lung maturity in preterm fetus. Presented before the Society of Perinatal Obstetricians Annual Meeting. San Antonio, Texas, 1984.

32. Kazzi GM, Gross TL., Rosen MG, et al: The relatioship of placental grade, fetal lung maturity, and neonatal aulcome in normal and complicated pregnancies. Am J. Obstet. Gynecol 1984;148:54.

33. Tabsh KM: Correlation of real time ultrasonic placenta grading with amniotic fluid lecithin/sphingomyelin ratio. Am J. Obstet. Gynecol 1983; 145:504.

34. Destro F Calcagnile F, Ceccarello P: Placental grade and pulmonary maturity in premature f'etuses. J. Clin Ultrasound 1985; 13: 637.

35. Hislop A. Developmental biology of the pulmonary circulation. Paediat Respir Rev 2005;6(1):35-43.

36. Fuke S, Kanzaki T, Mu J, Wasada K, Takemura M, Mirsuda N, Murata Y. Antenatal prediction of pulmonary hypoplasia by acceleration time/ ejection time ratio of fetal pulmonary arteries by Doppler blood flow velocimetry. Am J Obstet Gynecol 2003;188(1):228-33.

37. Azpurua-Pardi H, Funai EF, Kleine M, Luo G, Buhimschi CS, et al. Acceleration/ejection time ratio in the fetal pulmonary artery as a predictor of fetal lung maturity. 18th World Congress on Ultrasound in Obstetrics and Gynecology. Ultrasound Obstet Gynecol 2008;32:267-8.

38. Kähler C, Schleussner E, Möller A, Seewald H-J. Doppler measurements in fetoplacental vessels alter maternal betamethasone administration. Fetal Diagn Ther 2004;19:52-7.

39. Cabral AC, Pereira AK, Rodrigues RL. Assessment of fetal pulmonary artery flow by Doppler echocardiography after antenatal corticoid therapy. Int J Gynecol Obstet 2006;92:257-9.

40. Sosa-Olavarría A, Gutiérrez- Montoya H, Díaz-Guerrero L, GiugniChalbaud G. Evaluación mediante flujometría Doppler del ducto arterioso, arteria pulmonar y venas pulmonares en gestaciones de segundo y tercer trimestre. PDTP 2003;15(4):162-74.

\section{Fuentes de financiamiento}

Este estudio ha sido autofinanciado por el autor.

\section{Conflictos de interés}

El autor declara no tener ningún conflicto de interés.

\section{Correspondencia:}

Alex Alberto Guibovch Mesinas

Dirección: Jr. Nicolás Alcázar 730 - Lima 21

Teléfono: (51) 95088769

Correo electrónico: alexguibovichmesinas@yahoo.es 